\title{
ASPECTOS CLÍNICOS DA INFUSÃO INTRAPERITONEAL EM BOVINOS
}

\author{
Wilmar Sachetin Marçal, André Hiroshi Quadros Watanabe
}

Universidade Estadual de Londrina - UEL, Departamento de Clínicas Veterinárias, Curso de Medicina Veterinária, Londrina, PR. Email: wilmar@uel.br

\section{RESUMO}

A manutenção e a correta concentração de íons são fatores essenciais para a homeostase nos animais. Algumas enfermidades como hipocalcemia, hipoglicemia, diarreia e outras, comumente acometem bovinos, levando a desequilíbrios hidroeletrolíticos e ácido básicos. Como método terapêutico para correção dessas alterações realiza-se a fluidoterapia. Esse trabalho tem como intuito avaliar a utilização da via intraperitoneal para a administração de soluções cristalóides e outros medicamentos, avaliando-a como uma via eficaz e segura para reidratação e terapêutica dos animais enfermos.

Palavras-chave: concentração de íons; desequilíbrios hidroeletrolíticos e ácido básicos; fluidoterapia; homeostase; método terapêutico.

\section{CLINICALS ASPECTS OF INTRAPERITONEAL INFUSION IN COWS}

\section{ABSTRACT}

The maintenance and the proper concentration of ions are essential for homeostasis in animals. Some diseases such as hypocalcemia, hypoglycemia, diarrhea among others, commonly affect cattle, leading to eletrolute an acid base imbalances. Fluid therapy is done as a therapeutic method. This paper is intended to evaluate the use of intraperitoneal administrations of crystalloid solutions and other drugs as an effective and safe way for rehydration therapy of the sick animals.

Keywords: concentration of ions; electrolytes imbalances and basic acids; fluid therapy; homeostasis; therapeutic method.

\section{INTRODUÇÃO}

Diversas enfermidades podem causar um desequilíbrio hidroeletrolítico e metabólico nos animais domésticos, alterando concentrações de íons e afetando a volemia. Por essa razão, a manutenção e a correta concentração de íons são fatores essenciais para a homeostase nos animais (ALONSO; REJAS, 2012). Em bovinos, mais especificadamente, as principais enfermidades causadoras desses transtornos são: hipocalcemia, hipoglicemia, acidose metabólica e diarreia, além de outras causas de desidratação.

Sendo assim, para que os parâmetros normais sejam reestabelecidos, tem-se a necessidade de realizar como procedimento terapêutico a fluidoterapia. Esta pode se dar por diferentes vias como a intraperitoneal, a intraóssea, a endovenosa, a oral, a subcutânea e a intramuscular. A via mais utilizada é a endovenosa, que exige monitoramento constante, baixa velocidade de infusão e alguns riscos, dependendo da solução veiculada, como por exemplo, o gluconato ou borogluconato de cálcio.

A infusão intraperitoneal pode ser de grande valia onde existem animais doentes, pois nem sempre o médico veterinário está próximo da localidade e os procedimentos de emergência precisam ser imediatos (CONSTABLE et al., 1996). Por essa razão, sistematizar um procedimento terapêutico de forma rápida significa salvar a vida dos animais enfermos e diminuir prejuízos. Desse modo, a infusão intraperitoneal, precedida de padrões antissépticos, torna-se uma valiosa alternativa terapêutica, minimizando o tempo de infusão e otimizando a convalescença de vários animais enfermos (CONSTABLE, 2003). O objetivo foi avaliar a viabilidade da administração de soluções cristalóides somadas a medicamentos diversos, como gluconato ou borogluconato de cálcio, cloreto de magnésio, complexos vitamínicos, ferro, iodo, dextrose e outros, por via intraperitoneal em bovinos, tanto adultos quanto bezerros, tendo em vista sua classe 
terapêutica, que inclui hidratação, estimulante e medicação de suporte.

Tanto na medicina humana quando na ciência veterinária a fluidoterapia é considerada muito importante, seja ela para reidratação do paciente ou para auxílio na aplicação de determinados medicamentos na tentativa de reverter quadros de hipocalcemia, hipoglicemia, toxemia da prenhez, e outras enfermidades.

Nos animais domésticos de grande porte, aproximadamente $60 \%$ do peso corpóreo no adulto e $80 \%$ no neonato consiste de água, que se encontra em constante movimento entre os compartimentos intracelular e extracelular (DEARO; REICHMANN, 2001a).

As vias de administração de fluidoterapia são a endovenosa, oral, subcutânea, intraóssea e intraperitoneal. A via subcutânea é pouco utilizada, principalmente em grandes animais, uma vez que um animal com desidratação média e que pese $500 \mathrm{Kg}$, pode precisar de até 65 litros de solução por dia, um volume muito grande para essas vias de administração (DEARO; REICHMANN, 2001b). Por motivos práticos, a fluidoterapia endovenosa em grandes ruminantes é pouco utilizada, uma vez que a mesma necessita de constante vigilância, para manter a integridade do sistema e suas conexões (catéter, equipo, frascos e tubos extensores), pois os animais tendem a mastigá-las. A fluidoterapia oral é muito utilizada em bezerros diarreicos, uma vez que tem baixo custo e não precisa estar em constante observação do animal, já que seu volume total é parcelado em 4 ou 5 vezes ao dia e a administração de uma parcela é rápida. A via oral é amplamente utilizada, pois a solução administrada não tem de ser estéril, tem maior viabilidade econômica e pode ser administrada em grandes volumes, não havendo necessidade de cuidados especiais e podendo ser fornecida de forma parcelada (LISBOA, 2004). Contudo, não é indicada em casos de desidratações moderadas e graves.

Não há relatos da utilização da via intraóssea em grandes ruminantes, provavelmente pelo fácil acesso venoso nesses animais.

Bezerros e bovinos adultos frequentemente necessitam de fluidoterapia na correção do equilíbrio acidobásico e eletrolítico, para prover nutrientes ou em tratamentos de choque hipovolêmico. Os sinais clínicos apresentados pelos animais podem nos dar informações da enfermidade em casos de impossibilidade de se fazer um exame laboratorial. Fraqueza muscular, bem como depressão e inapetência podem resultar de mudanças de $\mathrm{pH}$, da concentração de potássio e sódio, ou queda da concentração de cálcio e glucose (CONSTABLE, 1999).

As soluções mais utilizadas na fluidoterapia são as cristalóides e as colóides. As soluções cristalóides contém água, eletrólitos e/ou açúcares, e são as mais empregadas na fluidoterapia por seu baixo custo. Suas moléculas são capazes de entrar em todos os compartimentos corpóreos por serem permeáveis às membranas capilares (KIRBY; RUDLOFF, 2004). De modo geral, são utilizados quando se objetiva a correção de alterações de volume, de eletrólitos, dos níveis de energia e do equilíbrio ácido básico. As soluções colóides possuem alto peso molecular, e quando administradas aumentam a pressão coloidosmótica intravascular e, com isso, estimulam a passagem de fluido do espaço intra para o extracelular (DEARO; REICHMANN, 2001b).

As soluções mais usadas na Medicina Veterinária são os cristalóides por serem mais baratas. De modo geral, são utilizados quando se objetiva a correção de alterações de volume, de eletrólitos, dos níveis de energia e do equilíbrio ácido básico (DEARO; REICHMANN, 2001b).

Durante a administração rápida de fluidos por via intravenosa é necessário um acompanhamento constante do animal para evitar edema pulmonar. Se isso ocorrer o ruminante irá apresentar como sinais clínicos taquipnéia, aumento na profundidade da respiração, narinas dilatadas, edema de conjuntiva e raramente tosse úmida. Em caso de administração de cálcio por via intravenosa o mesmo deve ser feito de forma lenta (gota a gota). A reação normal do organismo consiste em aumento da força de contração cardíaca com diminuição da frequência. Se a administração for feita de forma rápida pode causar arritmia e morte por fibrilação ventricular. A auscultação do coração do animal é indispensável durante o tratamento para evitar que o animal receba uma dose tóxica e letal de cálcio, já o monitoramento visual do pulso da jugular na base do pescoço não permite uma detecção precoce de bradicardia (CONSTABLE, 1999).

Em estudos, Silanikove (1991) mostrou que durante a reidratação de pequenos ruminantes por via intraperitoneal, $96 \%$ da 
quantidade infundida foi retida pelo organismo, enquanto que por via intravenosa, apenas $90 \%$ foi retida. Os parâmetros sanguíneos após a reidratação em ambos os tratamentos foram muito similares.

De acordo com Constable (2003), na fluidoterapia por via intraperitoneal podemos usar tanto fluidos colóides como cristalóides. $\mathrm{Na}$ fluidoterapia endovenosa podemos ter falhas na punção, na manutenção do acesso e problemas de arritmia ou bradicardia. Assim, a via intraperitoneal se torna uma opção muito mais segura e viável, principalmente em locais com pouca estrutura e mão de obra para que se possa realizar a contenção do animal.

A infusão intraperitoneal também pode ser de grande valia onde há um animal doente, porém o veterinário ainda irá demorar um pouco a chegar, visto que se trata de uma via de fácil acesso, e se feita uma boa antissepsia do local, há poucas chances de ocorrer algo errado.

\section{MATERIAL E MÉTODOS}

A avaliação foi realizada em bovinos provenientes de rebanhos particulares, com discreta apatia e manutenção de estação, presença de reflexo de sede e apetite, discreta diminuição da elasticidade da pele, mucosas rosadas pouco pegajosas, tempo de preenchimento capilar (TPC) variando de 2 a 4 segundos, sinais esses típicos de desidratação moderada. Portanto, para a realização desta terapêutica os autores trabalharam com bovinos naturalmente enfermos, não experimentais, sem submetê-los a desafios e/ou maus tratos, com aprovação pelo Comitê de Ética no Uso de Animais (CEUA-UEL), sob número 12717.2015.22.

O preparo dos produtos comerciais seguiu a recomendação do fabricante. As propriedades rurais situavam-se na região de Londrina, entre $23^{\circ} 08^{\prime} 47^{\prime \prime}$ e $23^{\circ} 55^{\prime} 46^{\prime \prime}$ de Latitude Sul e entre $50^{\circ} 52^{\prime} 23^{\prime \prime}$ e $51^{\circ} 19^{\prime} 11^{\prime \prime}$ a Oeste de Greenwich e 585 metros de altitude, norte do Paraná, Brasil (LONDRINA, 2014). Através do atendimento clínico foi possível avaliar a infusão intraperitoneal em 49 bovinos, sendo 41 vacas e oito bezerras, que apresentavam diarreia, pêlo eriçado e sem brilho, fraqueza, caquexia e anorexia. Nas vacas, o critério clínico de utilização foi hidratação pósparto, além de aplicações com soluções energéticas e vitaminas, visando melhor e mais rápida recuperação, em função do estado de enfraquecimento.

As infusões foram realizadas com soluções cristalóides (Ringer com lactato, solução fisiologia e solução glicosada a 5\%) em volumes que variaram de 1 a 2 litros em bezerras e de 2 a 5 litros em vacas adultas, somadas de complexos vitamínicos como B12 e E (20 a 40ml), protetores hepáticos (20 a 100ml), borogluconato de cálcio (20 a $100 \mathrm{ml})$, glicose $50 \%$ (20 a $50 \mathrm{ml}$ ) e iodeto de sódio $(20 \mathrm{ml})$.

O acesso intraperitonal, dar-se-á pela fossa paralombar do lado direito (Figura 1),

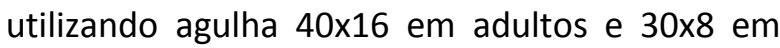
bezerros, acoplada a um equipo macro, após correta antissepsia do local com iodo degermante, álcool $70 \%$ e éter. A agulha é posicionada no meio da fossa paralombar e sua inserção ocorre num ângulo de $90^{\circ}$ em relação ao solo, ultrapassando a pele, o subcutâneo e os músculos oblíquo abdominal externo, oblíquo abdominal interno e transverso do abdômen, fáscia e peritônio, chegando assim, a cavidade abdominal. De acordo com Braun et al. (2011), a espessura da pele junto com os músculos do abdômen nas vacas adultas varia de 2,80 a $3,40 \mathrm{~cm}$; a pele e os tecidos do subcutâneo medem de 0,60 a $0,80 \mathrm{~cm}$, o músculo oblíquo abdominal externo 0,50 a $0,70 \mathrm{~cm}$, o oblíquo abdominal interno 0,50 a $0,70 \mathrm{~cm}$, o músculo transverso do abdômen de 0,50 a $0,75 \mathrm{~cm}$ enquanto que, as fáscias musculares e o peritônio medem no total de 0,20 a $0,30 \mathrm{~cm}$. 


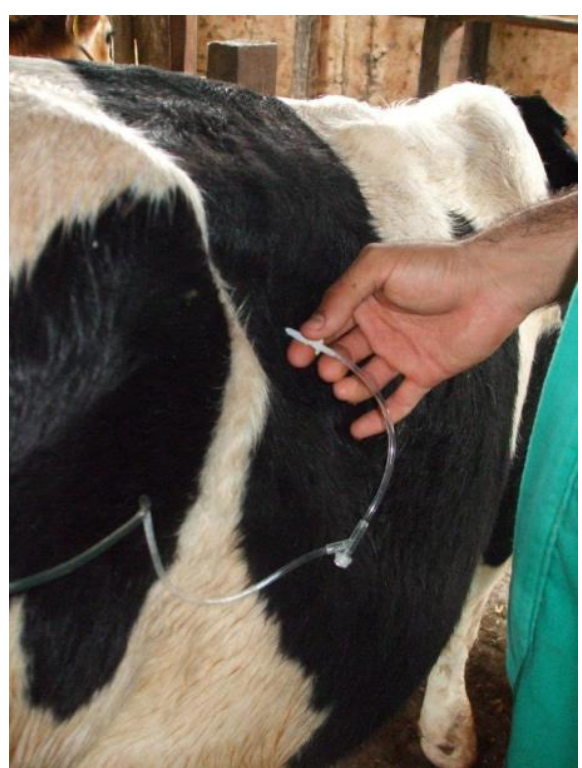

Figura 1. Agulha colocada na fossa paralombar e administração de fluidoterapia

Foi analisada a resposta do animal durante a administração da fluidoterapia a fim de detectar a ocorrência de arritmias, edema pulmonar ou outras alterações não desejáveis. Houve monitoração constante por meio de observação clínica e comportamental, além da auscultação pulmonar e cardíaca. Após duas semanas os animais eram avaliados novamente quanto à melhora do quadro, presença de alterações comportamentais e clínicas.

\section{RESULTADOS E DISCUSSÃO}

Foi possível comprovar a segurança da administração de medicamentos que contenham gluconato ou borogluconato de cálcio, cloreto de magnésio e dextrose, complexos vitamínicos entre outros, associados a soluções cristalóides (Ringer com lactato, solução fisiológica ou solução glicosada a 5\%) por via intraperitoneal em bovinos sem causar problemas como arritmias ou edemas pulmonares, mesmo em concentrações mais elevadas. Esses resultados estão em consonância com os observados por Kirby e Rudloff (2004).

Não houve formação de abscessos ou ocorrência de miíases na região perfurada, nem foi diagnosticado quadros de peritonites, de acordo com o que observaram também Braun et al. (2011).

Não se observou também, qualquer edema ou aumento de volume subcutâneo na região da infusão durante a mesma, havendo pronta recuperação dos animais após as infusões, retornando prontamente ao pastoreio e ingestão de água.
Nas fazendas produtoras de leite, principalmente nas pequenas propriedades, é muito comum não haver a presença constante de um médico veterinário nos momentos em que surgem as enfermidades. Muitas vezes um animal adoece e acaba vindo a óbito, pois o médico veterinário não consegue chegar a tempo e o tratador dos animais não tem capacidade paraum tratamento de suporte, se não for treinado.

Tendo em vista esses aspectos, a confirmação de uma via de acesso para fluidoterapia, que não causará danos a vasos importantes do sistema circulatório, poderá facilitar o trabalho do médico veterinário e abrirá uma nova possibilidade de administração de determinados produtos, podendo ser manuseados por capatazes e/ou auxiliares, de maneira segura e eficaz. Essa alternativa foi avaliada com sucesso por outros pesquisadores em tempos anteriores (CONSTABLE, 2003).

Como alguns procedimentos veterinários requerem urgência, a infusão intraperitoneal pode ser uma alternativa viável para o tratamento de animais que necessitam de um cuidado imediato em fazendas onde a presença do médico veterinário não é constante. Ao instituir um procedimento terapêutico de forma rápida e que obedeça aos padrões de antissepsia, tem-se por objetivo minimizar o tempo de infusão, melhorar a convalescença do animal e diminuir os prejuízos econômicos.

\section{CONCLUSÃO}

A infusão intraperitoneal em bovinos, através do presente experimento, demonstrou 
ser uma via rápida e segura de fluidoterapia, promovendo ganho de tempo nos serviços das fazendas, sem dificuldades operacionais.

\section{REFERÊNCIAS}

ALONSO, D.A.J.; REJAS, L.J. Fluidoterapia práctica em rumiantes. Disponível em: <http://www.veterinaria.org/recistas/recvet/n07 0708.html>. Acesso em: 6 mar. 2012.

BRAUN, U; GORBER, U; HÄSSIG, M; NUSS, K. Ultrasonography of the abdominal wall before and after laparotomy in cows. Schweizer Archiv für Tierheilkunde, v.153, n.2, p.71-77, 2011. http://dx.doi.org/10.1024/0036-7281/a000153 CONSTABLE, P.D.; GOHAR, H.M.; MORIN, D.E.; THURMON, J.C. Use of hypertonic saline-dextran solution to resuscitate hypovolemic calves with diarrhea. American Journal of Veterinary Research, v. 57, n.1, p.97-104, 1996.

CONSTABLE, P.D. Hypertonic saline. Veterinary Clinics of North America: Food Animal Practice, v.15, n.3, p.559-585, 1999. http://dx.doi.org/10.1016/S0749-0720(15)30164$\mathrm{X}$

CONSTABLE, P.D. Fluid and electrolyte Therapy in ruminants. Veterinary Clinics of North America: Food Animal Practice, v.19, p.557-597,2003. http://dx.doi.org/10.1016/S0749-0720(03)000549

DEARO, A.C.O.; REICHMANN, P. Fluidoterapia em grandes animais - Parte I: água corpórea, indicações e tipos de fluidos. Revista de Educação Continuada em Medicina Veterinária. CRMV-SP, São Paulo, v.4, n. 2, p.3-8, 2001a.

DEARO, A.C.O.; REICHMANN, P. Fluidoterapia em grandes animais - Parte II: Quantidades e vias de administração. Revista de Educação Continuada em Medicina Veterinária. CRMV-SP, São Paulo, v.4, n. 3, p.3-11, 2001b.

KIRBY, R.; RUDLOFF, E. Terapia de líquidos e eletrólitos. In: ETTINGER, S.J.; FELDMAN, E.C. Tratado de medicina interna veterinária. 5 . ed. São Paulo: Guanabara Kooga, 2004. p.342.

LISBOA, J.A.N. Fluidoterapia em ruminantes: uma abordagem prática. 2004. Disponível em: <http://www.spmv.org.br/conpavet2004/palestr a\%20-\%20resumos/julio\%20lisboa>. Acesso em: 15 dez. 2011.

LONDRINA. PREFEITURA MUNICIPAL. Portal da Prefeitura Municipal de Londrina. 2014. Disponível em: $<$ http://www.londrina.pr.gov.br./index.php?optio n=com_content\&view=article\&id=1251\%3Aacidade\&catid $=5 \% 3 \mathrm{Aa}$ - cidade\&ltemid=4\&showall=1>. Acesso em: 08 out. 2014.

SILANIKOVE, N. Effects of oral, intraperitoneal and intrajugular rehydrations on water retention, rumen volume, kidney function and thirst satiation in goats. Comparative Biochemistry Physiology, v.98A, n.2, p.253-258,1991. http://dx.doi.org/10.1016/0300-9629(91)90529-L

Recebido para publicação em 15/12/2015

Revisado em 07/06/2016

Aceito em 17/06/2016 